\title{
Identification of Modal Parameters for Reinforcement of Walls in Earthen Ruins
}

\author{
Bei Liu $\left(\mathbb{D},{ }^{1}\right.$ Yucheng Shi $\mathbb{D}^{1},{ }^{1}$ Kun Liu ${ }^{D},{ }^{2}$ Tao Li, ${ }^{1}$ and Shaopeng Wang ${ }^{1}$ \\ ${ }^{1}$ Lanzhou Institute of Seismology CEA, Lanzhou 730000, China \\ ${ }^{2}$ Lanzhou Institute of Geotechnique and Earthquake CEA, Lanzhou 730000, China \\ Correspondence should be addressed to Yucheng Shi; shiyc@gsdzj.gov.cn
}

Received 8 October 2021; Revised 28 October 2021; Accepted 1 November 2021; Published 18 November 2021

Academic Editor: bingxiang yuan

Copyright ( 2021 Bei Liu et al. This is an open access article distributed under the Creative Commons Attribution License, which permits unrestricted use, distribution, and reproduction in any medium, provided the original work is properly cited.

The protection of earthen sites plays an important role in the context of preservation of cultural heritage, especially in the inheritance and promotion of history and culture. The aim of the paper is to present the essential results of an ongoing research on a reinforced rammed earthen wall in Suoyang City (Guazhou, China). The wall vibrations caused by ambient actions were analyzed using the stochastic subspace algorithm to estimate the modal parameters of the wall. The frequencies of the first three orders are $3.566 \mathrm{~Hz}, 5.003 \mathrm{~Hz}$, and $6.250 \mathrm{~Hz}$, and the corresponding modes are first-order transverse bending, second-order left and right torsion, and third-order vertical bending, respectively. Then, according to the data of elastic modulus obtained in the lab, the finite element calculation is carried out, and referring to the results of field measurement, the revised elastic modulus value is $205.90 \mathrm{MPa}$. It is worth mentioning that the revised value is significantly improved from the original laboratory value, and it is also indicated that the seismic performance of the reinforced wall has been significantly improved. The present work is expected to provide a theoretical basis for reinforcement, protection, and seismic control of earthen ruins.

\section{Introduction}

Modal analysis is a crucial method in structural dynamics [1]. The interest on ancient architectural sites has increased the need for characteristics of the structures in these sites. Vibration tests are often performed on the structures to identify the modal characteristics, frequency, damping, and mode of vibration. As a kind of cultural relic, earthen ruins enjoy a long history and splendid culture [2]. Due to historical reasons, natural environment, and human factors, the structures in earthen ruins were vulnerable to plenty of diseases, such as sheet spalling, hollowing, fissure joints, washouts, and biological damage, which are likely to result in destruction of earthen sites. Therefore, the conservation of earthen sites is imminent [3]. Structural dynamic methods have been introduced to the analysis of earthen sites. Li et al. and Liu et al. conducted pulsation tests on the walls of earthen sites and obtained the spectral characteristics of the walls and self-oscillation behaviors $[4,5]$. Although they gained some structural properties for earthen sites, their data for analysis were obtained from one specific point, i.e., from the bottom or the top of the site walls, whereas the structural properties for one single point of the wall often differed from the those of the whole wall. Meng et al. studied the effect of microvibrations generated by vehicle loads on a bell tower in Xi'an by installing acceleration sensors in different locations [6]. There are also works on modal parameter identification of site buildings. For instance, Diaferio et al. performed an ambient excitation modal test analysis and modal parameter identification for the historic tower in Bari, Italy, and evaluated the bending modal vibration patterns for the first two orders [7, 8]. Aguilar and Ramos performed an ambient excitation modal test for a historic building in Peru to identify the dynamic characteristics of the building [9]. Conte et al. conducted structural identification on historical masonry buildings in southern Italy through field investigations, dynamic tests, and numerical simulations to ensure the dynamic response performance under the operational conditions of the building [10]. In summary, operational modal analysis and parameter identification are mostly applied to building structures but rarely to the reinforced walls of 
earthen site structures. Due to the low cost and the local availability of the soil material, it is widely used in architecture [11]. The rammed earth walls were widely constructed in the ancient times when science and technology were not developed for living and guarding. Rammed and granular soils differ substantially in structure. The soil should interact with other structures (plies) at the same time to reflect their structural behavior, while the soil itself cannot reflect the structural characteristics [12-15]. For example, Yuan et al. conducted the research in the field of the pile-soil interaction system [16], while the rammed earth structure can reflect its structural behaviors [17]. Therefore, the authors have reasons to think that it is feasible to apply operational modal analysis (OMA) to the earthen ruins. In order to better protect the earthen ruins, in other words, to increase its life span, various adverse factors need to be overcome, especially the damage caused by earthquake action [18]. It is necessary to study and analyze the structural behavior of soil sites. This shows the necessity of working modal analysis.

In the present work, a section of a reinforced wall in the Suoyang City architectural site is used as the research object. The measurement points are arranged simultaneously at the top and the bottom of the wall to collect data. The ambient excitation modal test and model fitting are performed by the stochastic subspace identification (SSI) method to obtain the frequencies and the vibration patterns of the first three orders. Finally, the equivalent elastic modulus of the reinforced wall is determined by combining the measurements with the result of finite element method (FEM) analysis.

\section{Methods and Procedures}

2.1. Overview of the Test Subject. As a national key protection unit of cultural relics, Suoyang City is a shining star in Chinese civilization. Under the influence of environmental and human factors, the earthen site in the ancient city has been damaged to varying degrees. Especially, it is prone to instant collapses during large earthquakes. Reinforcement is the most common means for protection of earthen ruins. The reinforced wall which was chosen as the test object is located at the northeast corner of the outer city of Suoyang. The condition of the completed reinforcement is shown in Figure 1. The wall is trapezoidal in cross section with the lengths of two sides being $6.5 \mathrm{~m}$ and $4.2 \mathrm{~m}$. It is $10 \mathrm{~m}$ high, and the thickness of the ramming layer is $0.1 \mathrm{~m}$. The upper surface of the site is roughly terrace-shaped. For better description, from left to right, they were named as a, b, and c, as shown in Figure 2. The first part (a) is about $9.6 \mathrm{~m}$ long. The second part (b) is a sloping surface with a length of $3.8 \mathrm{~m}$. The third part (c) is about $6.2 \mathrm{~m}$ long. The height difference between a and $\mathrm{c}$ is about $1 \mathrm{~m}$. The bottom of the measured surface is about $6 \mathrm{~m}$ long, and the rest is covered by collapsed soil, which shows an inclined plane, as shown in Figure 1.

\subsection{Experimental Measurements}

2.2.1. Instruments and Equipment. The main test instrument includes an INV 3060S intelligent signal acquisition and processing system. The highest sampling frequency was set at $51.2 \mathrm{kHz}$, and the sample analysis accuracy was 24-bit A/ D. The conventional amplitude error is less than $1 \%$, and the frequency error is less than $0.01 \%$. The vibration pickup sensors were selected for the 941B ultra-low frequency vibrometer developed by the Institute of Engineering $\mathrm{Me}$ chanics of the China Earthquake Administration. It is mainly used for pulsation measurement of the ground and structures and is suitable for testing the structures of earthen sites with low frequencies. The sensor has four modes: acceleration, small velocity, medium velocity, and large velocity. The mode selected for this test is small velocity, with a sensitivity of $23 \mathrm{~V}-\mathrm{s} / \mathrm{m}$, a maximum displacement range of $20 \mathrm{~mm}$, and a maximum velocity range of $0.125 \mathrm{~m} / \mathrm{s}$.

2.2.2. Procedures for Field Testing. As the test object is an earthen site and it is not suitable for the input external excitation method, the multiple-input multiple-output (MIMO) method was employed for the tests. The ground pulsation acted as the vibration source during the test.

The test was carried out in two groups. The first group was named measurement point $\mathrm{A} 1$ and point 1 , and the second group was named measurement point A1 and point 2. Point A1 is located at the bottom of the wall, which is defined as the reference point. The location of point 2 is shown in Figure 3. The arrangement of the overall measurement points is shown in Figure 4. Each measurement point was installed with three sensors along three directions: the wall direction, vertical wall direction, and vertical direction. All of them were placed on the surface of the wall. Each group was tested three times, and each test lasted 300 seconds, with a sampling frequency of $204.8 \mathrm{~Hz}$.

2.3. Modal Parameter Identification. The response signal of each measurement point was obtained through the test. The modal parameters can generally be identified in the time domain and frequency domain. The stochastic subspace identification (SSI) algorithm is often used for analysis in the time domain, and the enhanced frequency-domain decomposition (EFDD) method is used for analysis in the frequency domain [19]. SSI algorithm is a relatively mature linear system identification method, which can effectively obtain modal parameters from the structural response of environmental excitation. The stable graph method is a novel method to identify the order of the system. The modal identification was performed, applying the data-driven SSI algorithm available in the commercial software DASP. Application of the software to analyze the parameters was obtained by the SSI algorithm. On account of the denser modal frequencies, the identification accuracy with the EFDD was not precisely enough to identify the frequency range of interest, so the modal parameter identification was herein adopted by SSI algorithm.

The SSI algorithm was used to fit the modal data of test No. 1. The stability diagrams are shown in Figure 5. Combined with the vibration patterns, the first three orders of the modality of the earthen site structure were obtained. The modal frequencies and damping are shown in Table 1. The vibration patterns corresponding to each order of 


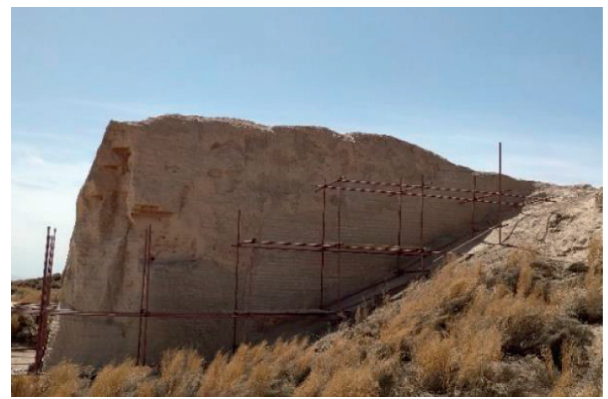

Figure 1: The reinforced site wall.

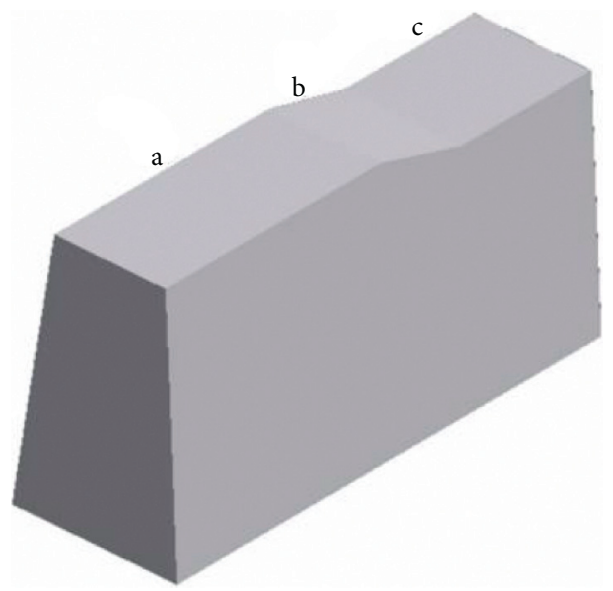

Figure 2: The simplified model of the wall.

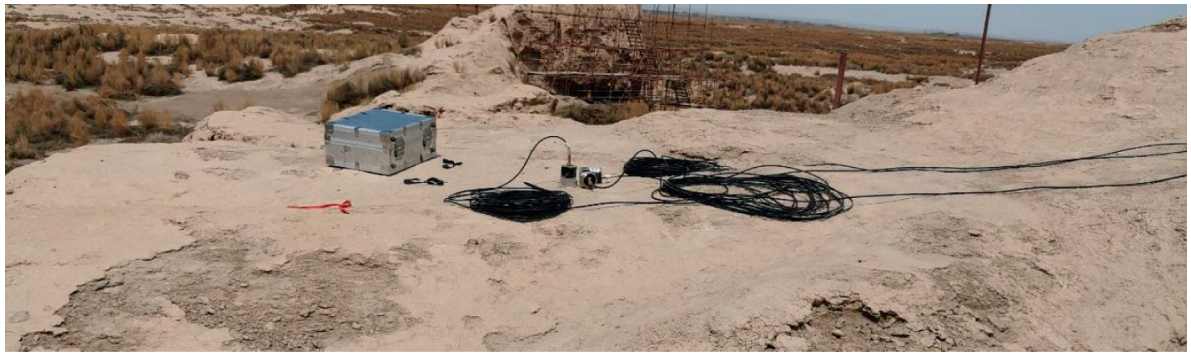

FIGURE 3: Measurement point 2.

frequencies were extracted by the DASP software [20]. The modal vibration patterns are shown in Figure 6. From the diagram of the vibration mode (Figure 6), it can be learned that the first-order vibration mode is the transverse bending mode in the $x$ and $y$ planes, the second-order vibration mode is the left and right torsional bending mode in the $x-y$ plane, and the third-order vibration mode is the vertical bending mode in the $y$ - $z$ plane. The data of tests No. 2 and No. 3 were obtained by following the same workflow. Table 1 shows the averages of the three results.

\section{Interpretation of the Results}

The characteristic parameters, including frequency, damping, and mode of vibration, were obtained by field testing and data processing. Table 2 summarizes the frequencies and the damping. The modes of the vibration are shown in Figure 7. The table clearly shows the fine distinction between the three tests. The accuracy of the frequencies can be verified among the three sets of data. However, the damping acquired from these data differed greatly which suggested that there is no good consistency. Finally, the final frequencies are determined by taking the average value. The next step is to analyze the parameters of the material which has the most significant effect on the structural properties. The main parameters affecting the structural characteristics are mass, elastics modulus, damping, boundary conditions, and state of conservation [22]. In order to select the parameters to evaluate the reinforcement effect better, the above parameters are briefly introduced below. The most common disease in earthen ruins is cut out, which will reduce the overall mass of soil sites, but the proportion is 


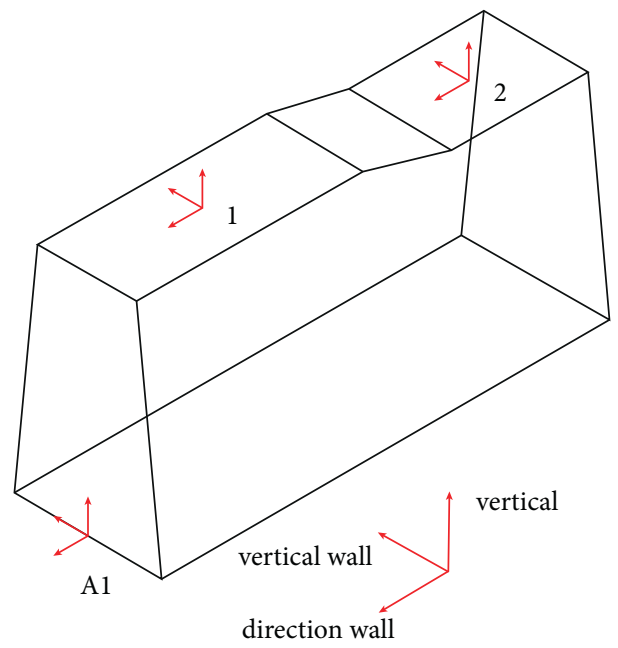

Figure 4: Distribution of measurement points. There are three measuring points in the model, and three sensors are placed at each point to measure three different directions.

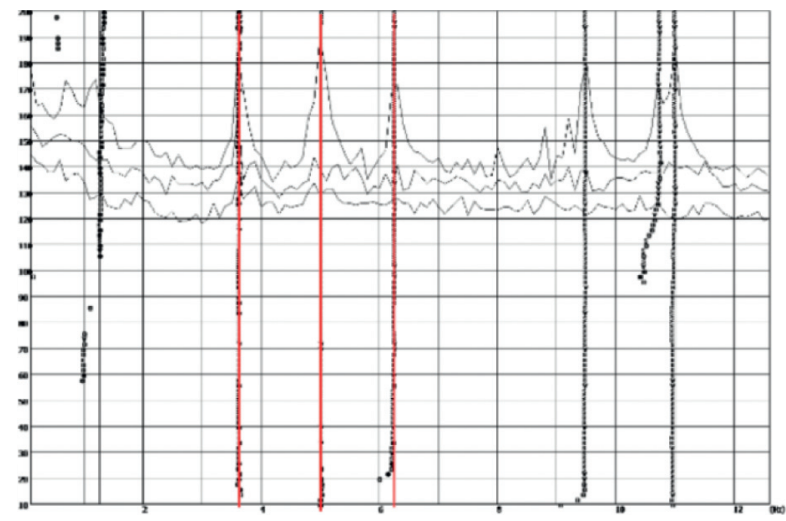

(a)

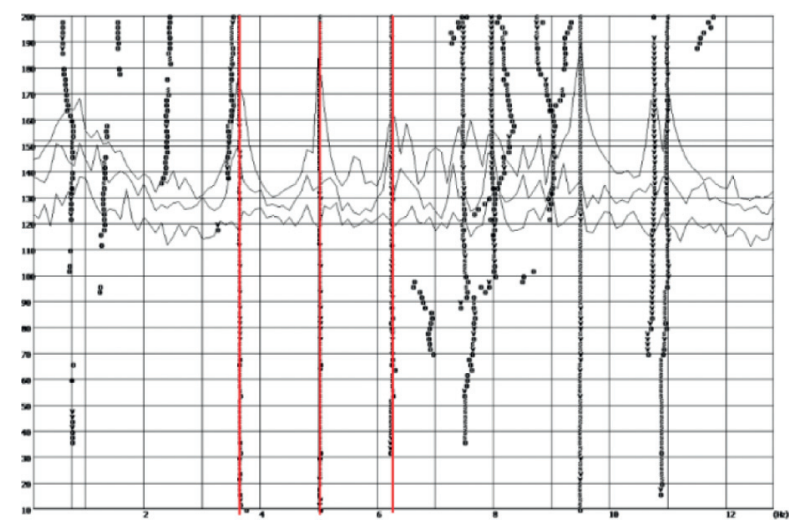

(b)

Figure 5: The stability of test No. 1. The red line represents the location of the identified frequencies. (a)The stability diagram of the first set of data. (b) The stability graph of the second set of data.

TABLE 1: Modal frequencies and damping of the first three orders of the reinforced wall.

\begin{tabular}{lcccccccc}
\hline \multirow{2}{*}{ Order } & \multicolumn{2}{c}{ Test No. 1 } & \multicolumn{2}{c}{ Test No. 2 } & \multicolumn{2}{c}{ Test No. 3 } & \multicolumn{2}{c}{ Mean } \\
& Freq (Hz) & Damp (\%) & Freq (Hz) & Damp (\%) & Freq (Hz) & Damp (\%) & Freq (Hz) & Damp (\%) \\
\hline 1 & 3.629 & 1.19 & 3.570 & 9.583 & 3.475 & 2.26 & 3.558 \\
2 & 5.012 & 0.82 & 4.997 & 4.246 & 4.999 & 0.55 & 5.003 \\
3 & 6.251 & 1.52 & 6.237 & 1.319 & 6.262 & 2.50 & 6.250 & 1.872 \\
\hline
\end{tabular}

small compared with the total mass. Elastic modulus is chosen in the paper. Different types of soil sites have different boundary conditions. However, the conditions are certainly for the specific objects. The same is true for the state of conservation. Let us go back to the basic theory. For the first mode, in order to simplify the model, the damping was neglected. We can apply it to a single-degree-of-freedom model, as shown in Figure 8. According to the first-order mode obtained above, the displacement occurs on the horizontal plane, and an undamped single-degree-of-freedom model is established [23], as shown in Figure 7. $m$ stands for concentrated mass, and support column is a massless uniform cantilever beam of bending stiffness $E I$. Under the condition of free vibration, the vibration equation of this model is

$$
\begin{aligned}
m \ddot{x}(t)+k_{e q} x(t) & =0, \\
K_{e q} & =\frac{3 E I}{L^{3}} .
\end{aligned}
$$

When the geometric size of the model is determined, that is, when $I$ and $L$ are constant in formula (1), the stiffness $K$ 


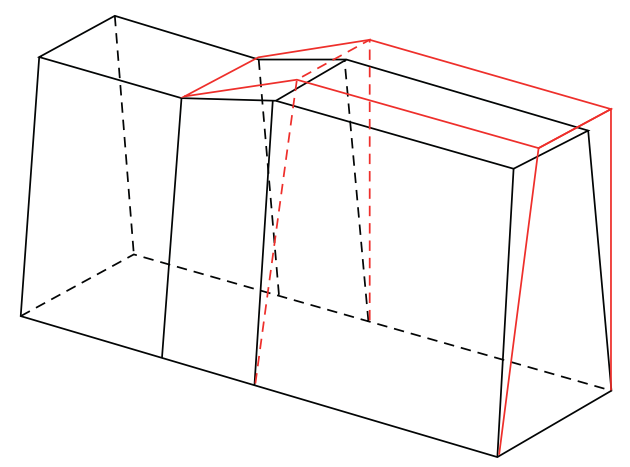

(a)

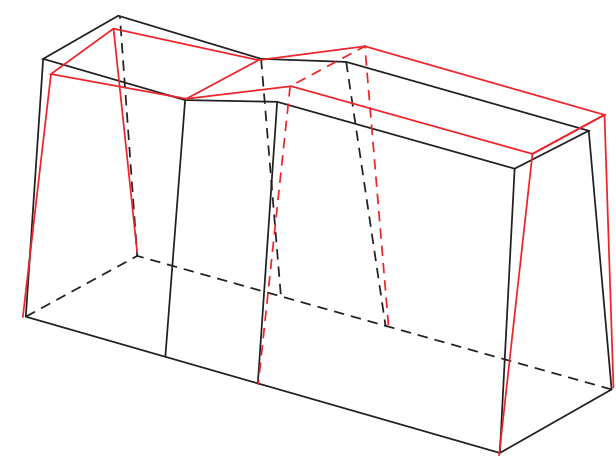

(b)

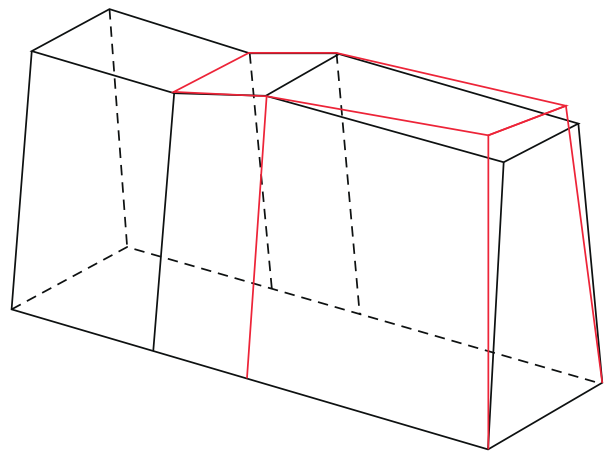

(c)

Figure 6: The first three orders of the mode of vibration. (a) First-order transverse bending. (b) Second-order left and right torsion. (c) Third-order vertical bending.

TABle 2: Physicomechanical parameters of materials [21].

\begin{tabular}{|c|c|c|c|c|c|}
\hline Material parameter & Density $\left(\mathrm{g} / \mathrm{cm}^{3}\right)$ & Cohesion $(\mathrm{kPa})$ & Angle of internal friction $\left({ }^{\circ}\right)$ & Poisson's ratio & $E(\mathrm{MPa})$ \\
\hline The wall & 1.86 & 24.3 & 31.2 & 0.3 & 136.09 \\
\hline
\end{tabular}

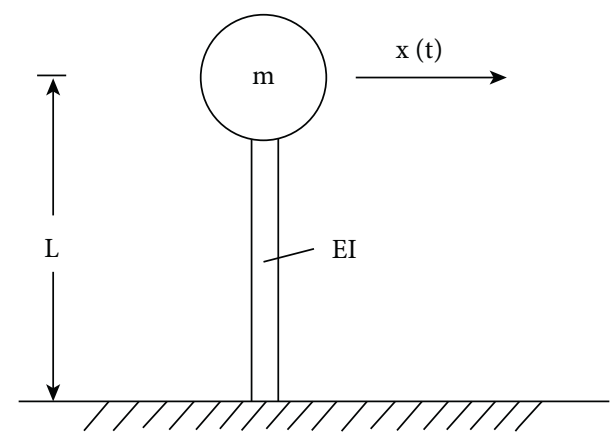

Figure 7: The single-degree-of-freedom model.

and $E$ are proportional to each other. That is the reason why $E$ is chosen as the evaluation of reinforcement effect in the paper.

In order to evaluate the reinforcement effect, the finite element method is needed. In recent years, the finite element method has been widely used [24]. The parameters to be analyzed are determined, and the finite element method is used for trial calculation. The parameters selected for finite element analysis are given in Table 2.
Figure 8 reveals the dimensions of the simplified model. By using the finite element method, we can obtain the first three modes of the simplified model. The frequencies are shown in Table 3, and the shapes of vibration are uncovered in Figure 9. Compared to the data obtained from the field tests, the frequency magnitudes obtained by the FEM are significantly smaller for all orders. However, the vibration patterns are consistent. The reasons are as follows: firstly, due to the special nature of the earthen site, the samples were 


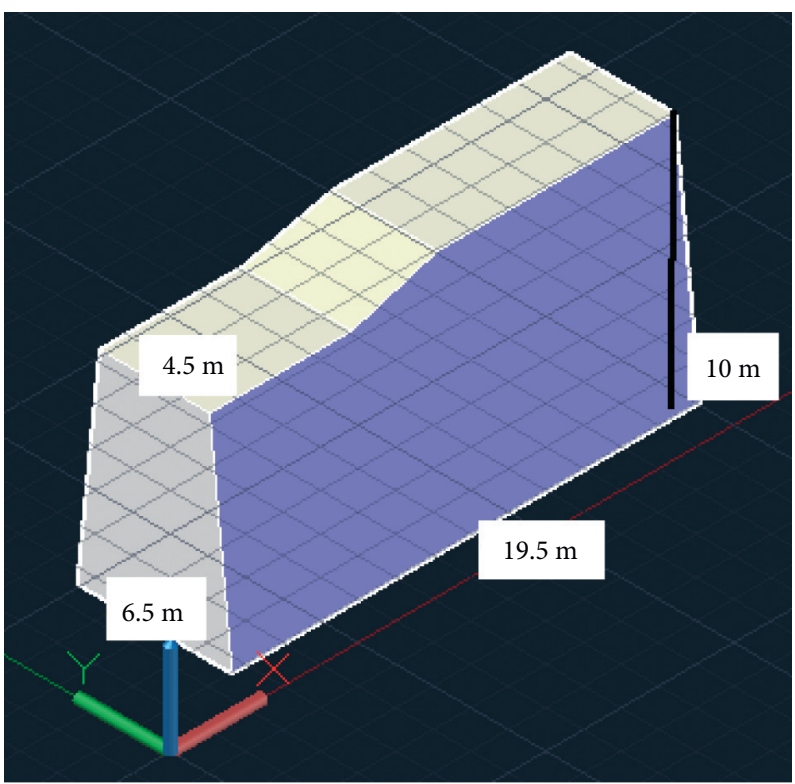

Figure 8: 3D model of the site wall.

TABle 3: The first three orders of modal frequencies for FEM.

\begin{tabular}{lcc}
\hline Order & Frequency $(\mathrm{Hz})$ & Mode of vibration \\
\hline 1 & 2.760 & First-order transverse bending \\
2 & 4.407 & Second-order left and right torsion \\
3 & 4.959 & Third-order vertical bending \\
\hline
\end{tabular}

selected from the blocks scattered around it and it was not possible to select from the earthen site itself, which would have damaged the site. This is something that cannot be tolerated as cultural heritage conservationists.

Secondly, there is deterioration of the soil samples due to climatic and other factors, which can further exacerbate the loss of strength of the soil and thus lead to small results [25]. At the same time, common sense agrees that the structural strength of the soil sites tested must have increased as a result of the treatment carried out. The next task to be undertaken is to modify the modulus of elasticity and to determine the final modulus of elasticity value with the aid of the FEM.

Existing works revealed that there is a certain functional relationship between the intrinsic frequency of the wall structure and the mass or stiffness of the structural material $[26,27]$ :

$$
f=K * \sqrt{\frac{E}{\rho}},
$$

where $E$ and $\rho$ denote the modulus of elasticity and density of the structure, respectively. For a given structure, $K$ can be considered as a constant.

The intrinsic frequency of the structure acquired from the experimental test is $\mathrm{fm}$. In the FEM, the elastic modulus value as the overall dynamic property of the wall site is determined with the help of $\mathrm{fm}$ and is defined as the equivalent elastic modulus $\mathrm{Em}$ for the reinforced wall site, which can reflect the health state of the structure after reinforcement. We assumed that the density and Poisson's ratio are constants [28]. From equation (2), it can be derived that

$$
C=\frac{f^{2}}{E}
$$

In the case of structure determination, $C$ is a constant, $f$ is the frequency of the structure, and $E$ is the modulus of elasticity of the structure. From (2) and (3), we can conclude that

$$
\frac{f_{F(i)}^{2}}{E_{0}}=\frac{f_{m(i)}^{2}}{E_{m i}},
$$

where $f F(i)$ denotes the $i$-th order frequency obtained using finite elements; $E_{0}$ denotes the starting modulus of elasticity; $f m(i)$ denotes the measured $i$-th order frequency; and $E_{m i}$ denotes the modulus of elasticity corresponding to the $i$-th order frequency.

The obtained results using the above method are shown in Table 4.

The results are shown in Table 5, where the frequencies of the test were compared with the result from finite element analysis using the equivalent elastic modulus. According to the results, the frequency errors of the first three orders are no larger than $10 \%$, so the equivalent elastic modulus is valid.

The modal parameters were identified using the SSI method, and the first three orders of modes were extracted: 

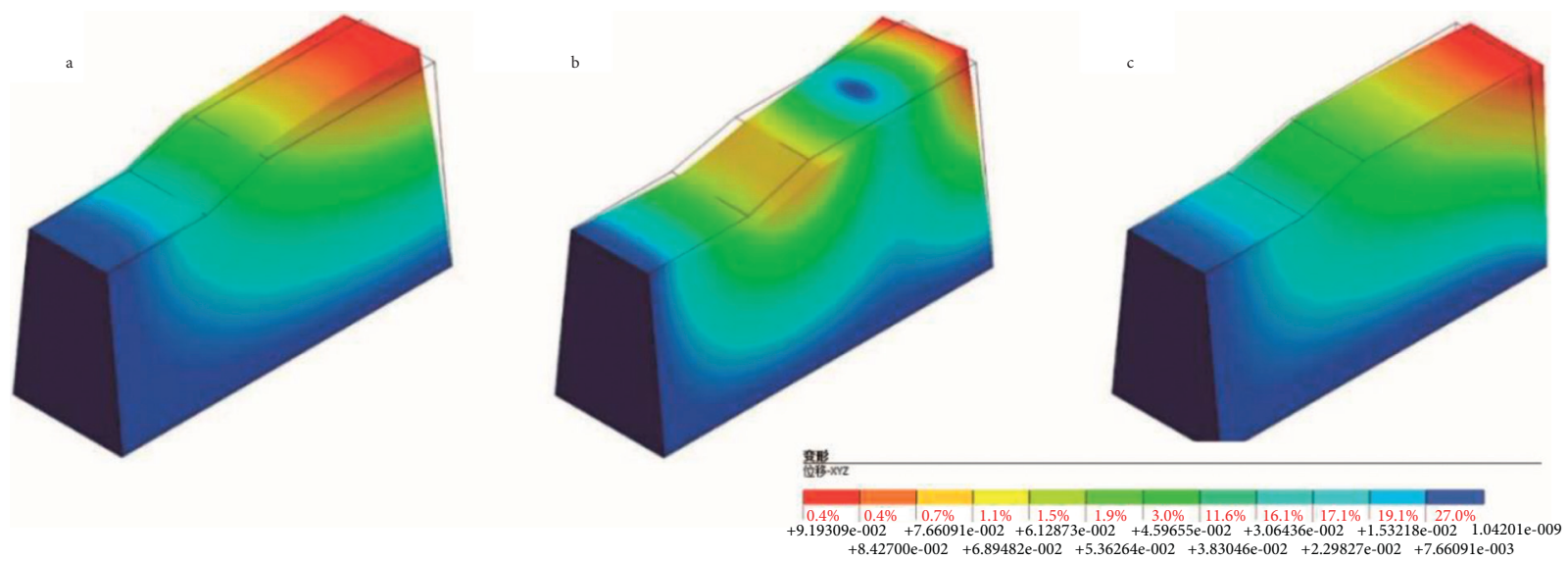

FiguRE 9: First three orders of modes of vibration. (a) First-order transverse bending. (b) Second-order left and right torsion. (c) Third-order vertical bending.

TABLE 4: The calculation results.

\begin{tabular}{lcccc}
\hline Order & $f_{F(i)}(\mathrm{Hz})$ & $f_{m(i)}(\mathrm{Hz})$ & $E_{m i}(\mathrm{MPa})$ & $E_{m}(\mathrm{MPa})$ \\
\hline 1 & 2.760 & 3.558 & 226.16 \\
2 & 4.407 & 5.003 & 175.38 & 205.90 \\
3 & 4.959 & 6.250 & 216.17 & \\
\hline
\end{tabular}

TABLE 5: The evaluation of the two methods.

\begin{tabular}{lccc}
\hline Order & Actual test $(\mathrm{Hz})$ & FEM $(\mathrm{Hz})$ & Error $(\%)$ \\
\hline 1 & 3.558 & 3.394 & 4.61 \\
2 & 5.003 & 5.417 & 8.38 \\
3 & 6.250 & 6.098 & 2.43 \\
\hline
\end{tabular}

the first-order mode was transverse bending at $3.558 \mathrm{~Hz}$; the second-order mode was left and right torsion at $5.003 \mathrm{~Hz}$; and the third-order mode was vertical bending at $6.250 \mathrm{~Hz}$. Also, the modes of vibration were extracted. By combining FEM results and the measured frequencies, the equivalent modulus of elasticity for the reinforced wall was obtained as 205.90 MPa. Compared to the initial modulus of elasticity, the structural characteristics of the wall have been significantly improved after reinforcement.

\section{Discussion}

The factors that influence the dynamic properties of the structure are the mass and the stiffness. The same is true for the modalities of the reinforced walls of earthen sites, which are the frequency, damping, and vibration pattern. Two aspects about the modulus of elasticity which impact the magnitude of the frequency and the vibration pattern will be discussed in the following.

On the one hand, the equivalent modulus of elasticity obtained is significantly larger than the initial value. To a certain extent, this reflects the effect of the treatment of the earthen site. The initial value parameters chosen in the paper are roughly close to those of the lime rammed earth wall in [21, 29]. It also demonstrates that the selected experimental samples do suffer from deterioration, and the degree of deterioration is roughly comparable to that of the lime rammed earth wall in the literature. It is the deterioration that reduces the strength of the structure. After the reinforcement treatment, the strength of the structure increases. But there is some difference with the data in Moein et al.'s article, with a difference of about $18.9 \%$ [29]. This is partly due to the choice of sample in the reference, which may have no or only slight deterioration of the soil, and partly due to the presence of cracks in the test object itself, which has a significant impact on the structural properties, especially in the interior [30]. Owing to the fact that the test object is in a pristine environment, it is also subject to external factors such as history and climate. Table 6 shows a comparison of the parameters between the two articles.

On the other hand, the vibration pattern diagrams revealed the locations of the displacement maxima when the structure is subjected to force. This also indicates that these are the points where damage is most likely to occur when subjected to external action. As the parameter studied in the paper is the modulus of elasticity, changes in the modulus of elasticity have no effect upon the modes. 
TABle 6: Comparison of the parameters between the articles.

\begin{tabular}{lcccc}
\hline Parameters & \multicolumn{2}{c}{ Reference [29] } & & This article \\
& RE & LSRE & Initial value & Final value \\
\hline$E(\mathrm{MPa})$ & 253.9 & 145.7 & 136.09 & 205.9 \\
Density $\left(\mathrm{g} / \mathrm{cm}^{3}\right)$ & 1.94 & 1.80 & 1.86 & 1.86 \\
\hline
\end{tabular}

\section{Conclusion}

(1) The modal parameters for reinforcement of walls in earthen sites were identified using the SSI method. The first three orders of modes were extracted: the firstorder mode was transverse bending at $3.566 \mathrm{~Hz}$, the second-order mode was left and right torsion at $5.003 \mathrm{~Hz}$, and the third-order mode was vertical bending at $6.250 \mathrm{~Hz}$. Also, the modes of vibration were extracted.

(2) Comparing the test data with the relevant literature, the modulus of elasticity values obtained from the laboratory are on the low side. Meanwhile, there is deterioration of the soil in the samples selected, as well as the way in which the samples are selected.

(3) The final equivalent modulus of elasticity obtained using the FEM is $205.9 \mathrm{MPa}$. The strength of the structure has been significantly improved after the strengthening treatment. However, it is still not up to that of a complete rammed earth wall.

(4) The change in modulus of elasticity does not affect its modal vibration pattern, but it can reflect the location of the structure where damage is likely to occur. Thus, it plays an important role in the analysis of its stresses and displacements.

\section{Data Availability}

Experimental data were obtained from field tests; some data were cited from references and annotated in the paper.

\section{Conflicts of Interest}

The authors declare that there are no conflicts of interest regarding the publication of this paper.

\section{Acknowledgments}

The authors acknowledge S. D. Wei from COINV for his support with test methods and software processing. The authors also wish to thank W. T. Tian, Y. X. Lu, and P. H. Chi for their contributions to this research. This study was financially supported by the National Key Research and Development Program of China (2020YFC1522200), through the project "The Study on Degradation Process and Protection Technology of Earthen Ruins under Multi-Field Coupled."

\section{References}

[1] L. Zhang, Modal Analysis and experiment, Tsinghua University Press, Beijing China, 2011.
[2] M. L. Sun, X. D. Wang, and M. X. Li, A Primer on the Conservation of Earthen Sites, Science Press, Beijing China, 2010.

[3] T. L. Li, Study on Reinforcement Methods of Earthen Sites and its Anti-seismic Stability, Lanzhou Institute of Seismology, Lanzhou, China, 2020.

[4] T. L. Li, Y. C. Shi, K. Liu, Y. X. Lu, C. Y. Wang, and Z. Y. Xian, "Disease characteristic and dynamic test analysis of Jiaohe ancient city," Journal of Seismological Research, vol. 43, no. 3, pp. 456-462+601, 2020.

[5] K. Liu, T. L. Li, Y. C. Shi, H. M. Tang, and Z. Y. Xian, "Dynamic response analysis of inner soil wall ruins in ancient Gao-Chang City," Journal of Disaster Prevention and Mitigation Engineering, vol. 40, no. 6, pp. 754-760, 2020.

[6] Z. B. Meng, Y. Z. Chang, L. Song, and J. Yuan, "The effects of micro-vibration excited by traffic vehicles on Xi'an bell tower," in Proceedings of the International Conference on Transportation Engineering, Chengdu, China, July 2009.

[7] M. Diaferio, "Dynamic analysis of a historical fortified tower," Key Engineering Materials, vol. 628, pp. 178-184, 2015.

[8] M. Diaferio, D. Foti, and N. I. Giannoccaro, "Non-destructive characterization and identification of the modal parameters of an old masonry tower," in Proceedings of the 2014 IEEE Workshop on Environmental, Energy and Structural Monitoring Systems, Proceedings, Naples, Italy, September 2014.

[9] R. Aguilar and F. Ramos, "Experimental modal identification of an existent earthen residential building," in Proceedings of the 5th International Operational Modal Analysis Conference (IOMAC 2013). Guimaraes, Portugal, 2013.

[10] C. Conte, C. Rainieri, M. A. Aiello, and G. Fabbrocino, “onsite assessment of masonry vaults: dynamic tests and numerical analysis," Geofizika, vol. 28, pp. 127-143, 2011.

[11] F. Greco and P. B. Lourenço, "Seismic assessment of large historic vernacular adobe buildings in the Andean Region of Peru. Learning from Casa Arones in cusco," Journal of Building Engineering, vol. 40, Article ID 102341, 2021.

[12] M. Sarcheshmehpour, M. Shabanlou, Z. Meghdadi, H. E. Estekanchi, and M. Mofid, "Seismic evaluation of steel plate shear wall systems considering soil-structure interaction," Soil Dynamics and Earthquake Engineering, vol. 145, Article ID 106738, 2021.

[13] L. E. Pérez-Rocha, J. Avilés-López, and A. Tena-Colunga, "Base isolation for mid-rise buildings in presence of soilstructure interaction," Soil Dynamics and Earthquake Engineering, vol. 151, Article ID 106980, 2021.

[14] J. Sucasaca and E. Sáez, "Topographical and structure-soilstructure interaction effects on dynamic behavior of shearwall buildings on coastal scarp," Engineering Structures, vol. 247, Article ID 113113, 2021.

[15] H. Long, Z. Wang, C. Zhang, H. Zhuang, W. Chen, and C. Peng, "Nonlinear study on the structure-soil-structure interaction of seismic response among high-rise buildings," Engineering Structures, vol. 242, Article ID 112550, 2021.

[16] B. Yuan, Z. Li, Z. Zhao, H. Ni, Z. Su, and Z. Li, "Experimental study of displacement field of layered soils surrounding 
laterally loaded pile based on Transparent Soil," Journal of Soils and Sediments, vol. 21, no. 9, pp. 3072-3083, 2021.

[17] R. A. Silva, O. Domínguez-Martínez, D. V. Oliveira, and E. B. Pereira, "Comparison of the performance of hydraulic lime- and clay-based grouts in the repair of rammed earth," Construction and Building Materials, vol. 193, pp. 384-394, 2018.

[18] P. Baquedano, R. R. Eudave, F. N. Miranda, T. M. Ferreira, and D. Gautam, "Traditional earth construction in Latin America: a review on the construction systems and reinforcement strategies," Masonry Construction in Active Seismic Regions, Woodhead Publishing, Sawston, UK, pp. 99-121, 2021.

[19] P. V. Overschee and B. D. Moor, Subspace Identification for Linear Systems: Theory-Implementation- Applications, Kluwer Academic Publishers, London, UK, 1996.

[20] R. J. Guo, G. X. Ma, and C. Wang, "Identification of modal parameters of large damped foundation structures," in Proceedings of the 26th National Conference on High Technology and Applications of Vibration and Noise, USA, June 2015.

[21] Y. C. Shi, M. Q. Hu, C. Wang, and Z. Y. Zhang, "A study of the response of the earthen archaeological sites during different seismic loadings," in Proceedings of the Eleventh National Conference on Rock Mechanics and Engineering, pp. 98-102, Wuhan,china, 2010.

[22] F. Lorenzoni, M. R. Valluzzi, M. Salvalaggio, A. Minello, and C. Modena, "Operational modal analysis for the characterization of ancient water towers in Pompeii," Procedia Engineering, Elsevier, vol. 199, , pp. 3374-3379, 2017.

[23] L. Meirovitch, Fundamentals of Vibrations, pp. 80-86, Waveland Press, Sydney, Australia, 2010.

[24] B. X. Yuan, Z. H. Li, Z. L. Su, Q. Luo, M. Chen, and Z. Zhao, "Sensitivity ofMultistage fill slope based onFinite element model," Advances inCivil Engineering, vol. 2021, Article ID 6622936, 13 pages, 2021.

[25] B. Yuan, Z. Li, Y. Chen et al., "Mechanical and microstructural properties of recycling granite residual soil reinforced with glass fiber and liquid-modified polyvinyl alcohol polymer," Chemosphere, vol. 286, Article ID 131652, 2022.

[26] Q. B. Bai, J. C. Morel, S. Hans, and N. Meunier, "Compression behaviour of non-industrial materials in civil engineering by three scale experiments: the case of rammed earth," Materials and Structures, vol. 42, pp. 1101-1116, 2009.

[27] A. De Sortis, E. Antonacci, and F. Vestroni, "Dynamic identification of a masonry building using forced vibration tests," Engineering Structures, vol. 27, no. 2, pp. 155-165, 2005.

[28] Y. L. Wang, A Theoretical Study on Structural Stability Assessment for Historical Ramparts in Dry Areas, Xi'an University of Architecture and Technology, Xi'an, China, 2018.

[29] R. Moein, E. Abolfazl, and R. Hamid, "Seismic performance of stabilised/unstabilised rammed earth walls," Engineering Structures, vol. 245, 2021.

[30] I. Arto, R. Gallego, H. Cifuentes, E. Puertas, and M. L. Gutiérrez-Carrillo, "Fracture behavior of rammed earth in historic buildings," Construction and Building Materials, vol. 289, Article ID 123167, 2021. 